\title{
Bilateral retinal hamartomas in neurofibromatosis type 2
}

William V Good, Michael C Brodsky, Michael S Edwards, William F Hoyt

Neuro-ophthalmology Unit, Departments of Neurologic Surgery,

Neurology, and Ophthalmology, University of California School of Medicine, San Francisco, CA 94143 W V Good M S Edwards W F Hoyt

\section{Department of} for Medical Sciences, Little Rock, Arkansas, USA

M C Brodsky

Correspondence to:

William V Good, MD, 400 Parnassus Avenue, A-704 San Francisco, CA 94143, USA.

Accepted for publication 6 September 1990 Ophthalmology, University of Arkansas

Abstract

We report a case of bilateral retinal hamartomas in a child with neurofibromatosis type 2 . This unique case demonstrates that a diversity of retinal changes can occur in this disease.

Neurofibromatosis (NF) consists of at least two genetically and clinically distinct types, NF-1 (von Recklinghausen's type) and NF-2 (acoustic neuroma type).' 'Gene defects on chromosome 17 are responsible for NF-1. A loss of genes on chromosome 22 causes NF-2.

Cotlier reported a combined pigment epithelial and retinal hamartoma (CPERH) in a patient who, in retrospect, had NF- $2 .^{2}$ Landau

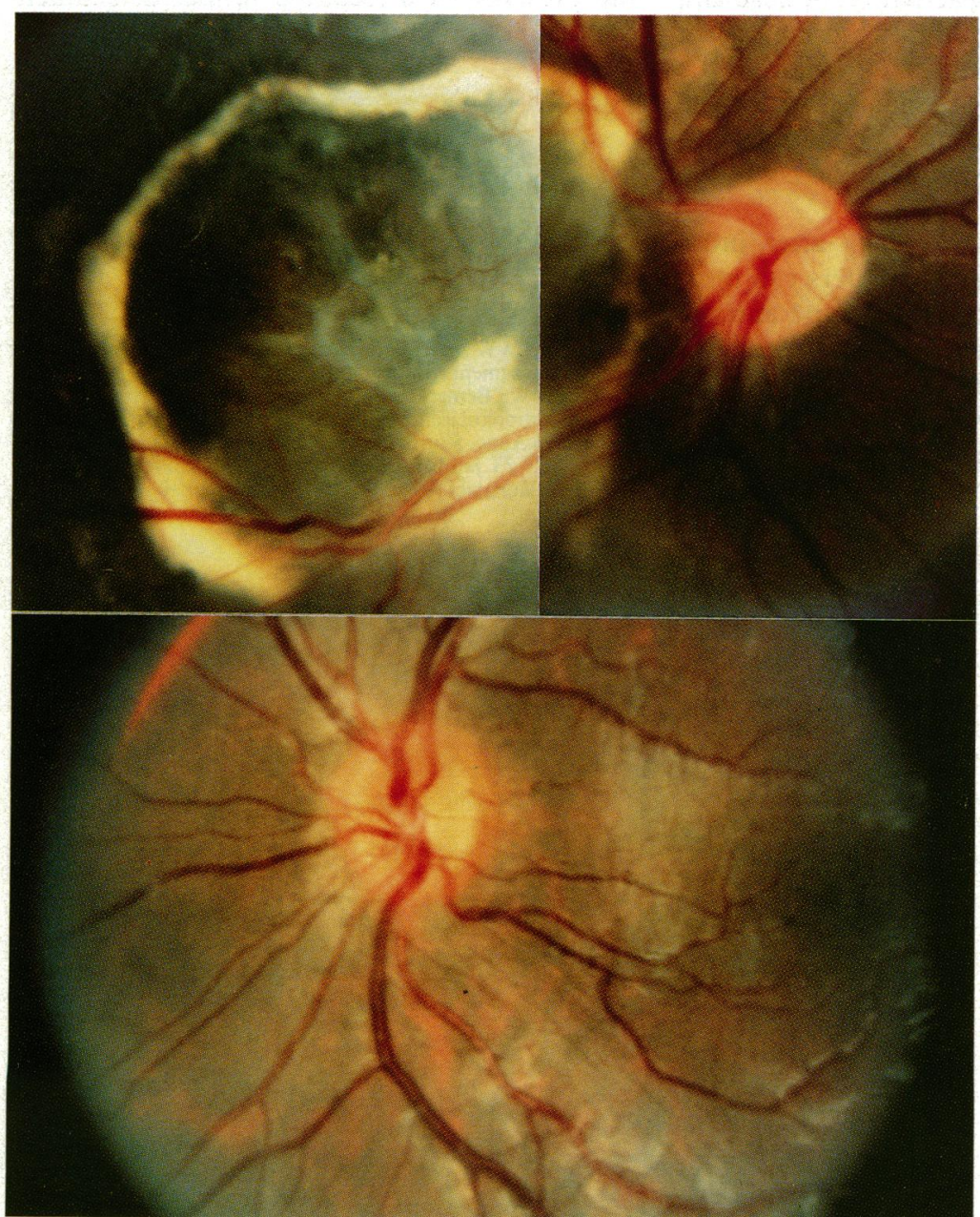

Figure 1 Above: Right eye, lesion involving optic nerve head and macula. Below: Left eye, areas of presumed glial proliferation on inner surface of retina. et al reported the possible association of the CPERH with NF-2 in another patient. ${ }^{3}$ We report another case of bilateral hamartomas in a patient with NF-2.

\section{Case report}

A 7-year-old boy was admitted to the University of California San Francisco Neurosurgery Service for evaluation of hemiatrophy and fasciculation of the right side of the tongue. He had one café-au-lait spot and diminished hearing on the right. His mother, grandmother, and two aunts were afflicted with neurofibromatosis and bilateral acoustic neuromas. A CT scan showed a partially calcified mass in the right cerebellopontine angle. At surgery a large meningioma of the cerebellopontine angle was subtotally resected. Histologically it was found to be malignant. Neurofibromas of cranial nerves 11 and 12 and a second meningioma extending through the hypoglossal canal were also noted.

Exotropia had been surgically corrected at age 2. Visual acuity was $20 / 800$ in the right eye and $20 / 20$ in the left. Right esotropia was present, with ductions full. In the right fundus (Fig 1) thre was a lesion involving the optic nerve head and macula. The lesion was bordered by a rim of subretinal fibrosis with a diaphanous glial membrane in its centre. Retinal vessels were pulled towards the lesion. The left retina contained multiple $1 / 4$ to $1 / 2$ disc diameter areas of presumed glial proliferation on the inner surface of the retina. These lesions were located inferotemporally and obscured underlying retinal vessels in some areas. They caused a distorted light reflex from the inner surface of the retina.

\section{Discussion}

Our patient had multiple cranial nerve tumours and meningiomas, and a family history of bilateral acoustic neuromas. He was afflicted with the genetic defect that causes NF-2. The CPERH lesions in NF-2 patients reported by Cotlier ${ }^{2}$ and by Landau $e t a l^{3}$ are similar. Our case is quite different and exemplifies a diversity of retinal changes that can be found in NF-2.

1 National Institute Health Consensus Development Conference Statement of Neurofibromatosis. Arch Neurol 1988; 45: 575-8.

2 Cotlier E. Café-au-lait spots of the fundus in neurofibromatosis. Arch Ophthalmol 1977; 95: 1990-2.

3 Landau K, Muci-Mendoza R, Dossetor F M, Hoyt WF Retinal hamartoma in neurofibromatosis 2. Arch Ophthalmol 1990; 108: 328-9. 